Rapport - Société canadienne d'histoire de l'Église catholique

\title{
Messire Jacques Paquin, curé de Saint-Eustache de la Rivière-du-Chêne (1821-1847)
}

\section{Louis-Joseph Rodrigue}

Volume 31, 1964

URI : https://id.erudit.org/iderudit/1007348ar

DOI : https://doi.org/10.7202/1007348ar

Aller au sommaire du numéro

Éditeur(s)

La Société canadienne d'histoire de l'Église catholique

ISSN

0318-6148 (imprimé)

1927-7075 (numérique)

Découvrir la revue

Citer cet article

Rodrigue, L.-J. (1964). Messire Jacques Paquin, curé de Saint-Eustache de la Rivière-du-Chêne (1821-1847). Rapport - Société canadienne d'histoire de l'Église catholique, 31, 73-83. https://doi.org/10.7202/1007348ar

Tous droits réservés @ La Société canadienne d'histoire de l'Église catholique, 1965
Ce document est protégé par la loi sur le droit d'auteur. L’utilisation des services d'Érudit (y compris la reproduction) est assujettie à sa politique d'utilisation que vous pouvez consulter en ligne.

https://apropos.erudit.org/fr/usagers/politique-dutilisation/ 


\title{
Messire Jacques Paquin curé de Saint-Eustache de la Rivière-du-Chêne (1821-1847)
}

\begin{abstract}
Le nom de Messire Jacques Paquin a déjà retenu l'attention de quelques historiens, dont le R.P. Thomas-Marie Charland, o.p. Ce travail, rédigé par l'un des successeurs du curé Paquin à Saint-Eustache, apporte une contribution plus modeste à l'histoire. Il se propose de présenter, à l'aide des documents paroissiaux et des archives diocésaines, un curé de paroisse aussi zélé que pittoresque dont le nom appartient — pour des raisons différentes de celles qu'il désirait - à la grande comme à la petite histoire.
\end{abstract}

Saint-Eustache de la Rivière-du-Chêne est la plus ancienne paroisse du comté des Deux-Montagnes. Les premiers actes de ses registres remontent à 1769. Après plusieurs desservants, dont le R.P. de Berrey, supérieur des Récollets de Québec, le premier curé résidant était nommé en 1778.

Au début d'octobre 1821 arrivait son quatrième curé, Messire Jacques Paquin. Il devait y demeurer vingt-six ans et y terminer sa carrière qui continue d'intéresser les amateurs de la petite et de la grande histoire, surtout à cause des événements qui se sont déroulés en 1837 dans le comté des Deux-Montagnes.

\section{Saint-Eustache de la Rivière-du-Chêne et le comté des Deux-Montagnes}

A cette époque, la paroisse de Saint-Eustache comprenait toute la seigneurie de la Rivière du Chêne — partie ouest de la seigneurie des Mille-Iles - soit deux lieues et un quart de front et trois lieues de profondeur. La famille Dumont possédait les deux tiers de ce territoire, dont celui du village; la famille de Bellefeuille possédait l'autre tiers dont la partie qui devait former, à partir de 1839, la paroisse de SaintAugustin. Le curé Paquin, dans sa première lettre adressée à $\mathrm{M}^{\mathrm{gr}}$ Plessis, prétendait que sa paroisse comprenait 6,000 âmes. Cette affirmation était certainement exagérée si nous comparons les chiffres fournis par le seigneur Dumont lui-même, dans une lettre à $\mathrm{M}^{\mathrm{gr}}$ Panet du 26 novembre 1829. Il y parle de 350 emplacements, dont 25 à 30 occupés par des protestants, un tiers du sol (de toute la paroisse) est inculte ou coupé en lopins de terre de si faible étendue que leurs propriétaires vivent à la journée n'ayant pas assez de leurs terres pour leur subsistance. Le bourg, centre du commerce et des affaires, comprenait à son dire 900 habitants. Les statistiques diocésaines compilées à l'occasion de la visite pastorale de 1838-1839 donnent pour Saint-Eustache une population de 3,000, dont 1,900 communiants, et de 2,661 pour Saint-Augustin, dont 1,600 communiants. Précisons que depuis 1834, les familles de la côte inférieure de Saint-Joseph, dans la seigneurie 
du Lac des Deux-Montagnes, avaient été annexées à la paroisse de Saint-Eustache.

Pour compléter ces données disons, en nous basant sur le Rapport du comité permanent de l'Education en 1829 , que la population totale du Bas-Canada, à l'époque, atteignait 511,919 habitants, que la population de la province était rurale dans la proportion de $85 \%$, que le comté d'York (Deux-Montagnes) comprenant les cantons de Chatham et de Grenville et la seigneurie d'Argenteuil, avec ses 20,905 habitants, était le comté le plus nonuleux du Ras-Canada. I.orsque Messire Jarqques Paquin deviendra archiprêtre, en 1833, son doyenné, en plus de SainteRose qui appartenait au comté voisin, comprenait, sur le territoire des Deux-Montagnes, les paroisses de Saint-Eustache, Saint-Benoît, SainteScholastique et Saint-Colomban.

\section{Messire Jacques Paquin}

Présentons maintenant dans ce cadre notre personnage. J'utilise ici une inscription qui figure au-dessous du portrait de Messire Jacques Paquin, dans la galerie historique du séminaire de Nicolet, où il se présente en brillante et nombreuse compagnie.

«L'abbé Jacques Paquin, né à Deschambault, comté de Portneuf, le 19 septembre 1791, de Paul Paquin et de Marguerite Marcotte, élève du Séminaire de Nicolet, de 1808 à 1813, fut ordonné prêtre le 24 septembre 1814. Vicaire à Varennes (1814-1815), curé à Saint-Françoisdu-Lac (1815-1821), de Saint-Eustache (1821-1847) où il fonda le couvent des Sœurs de la Congrégation en 1833, subit les malheurs des troubles de 1837 en cette paroisse et décéda le 7 décembre 1847. Archiprêtre, premier historien de l'Histoire de l'Eglise, missionnaire des Abénaquis pendant six ans, auteur du " Journal historique des Evénements arrivés à Saint-Eustache " et de "Mémoires sur l'Eglise du Canada ".

Voilà un curé qui ne risque pas de demeurer complètement ignoré. Aussi le R.P. Thomas-Marie Charland, o.p., dans un travail présenté à la Société canadienne d'Histoire de l'Eglise catholique, puis dans une conférence plus élaborée présentée à l'assemblée annuelle de la Société historique de Montréal, le 30 janvier 1946, a-t-il déjà fait connaître les côtés très pittoresques du personnage et réduit à ses justes dimensions le titre un peu ronflant de " premier historien de l'Histoire de l'Eglise » et d' " auteur de Mémoires (par ailleurs inédits, et pour cause !) de l'Eglise du Canada ». Le R.P. Thomas-Marie Charland, o.p., dans "Les Abénakis d'Odanak", publié tout récemment aux Editions du Lévrier (1964), a également traité des six années de vie missionnaire de Messire Jacques Paquin, comme curé de Saint-François-du-Lac et missionnaire des Abénakis.

En choisissant de vous présenter le curé de Saint-Eustache de la Rivière-du-Chêne j'ai voulu plutôt parcourir un peu à la hâte, j'en suis confus, quelques pages de nos archives paroissiales - documents, récits d'événements, livres de compte - relire avec vous quelques-unes des lettres contenues dans le dossier de Saint-Eustache (archives du dio- 
cèse de Montréal) et rendre hommage au zèle pastoral de l'un des curés d'il y a cent vingt-cinq ans.

\section{Saint-Eustache de la Rivière-du-Chêne en 1821}

Dès son arrivée à Saint-Eustache, le curé Paquin se rendit compte de l'ampleur de la tâche qui l'attendait. Il ne tarda pas à s'en ouvrir à son évêque, $\mathrm{M}^{\mathrm{gr}}$ Plessis, dans une longue lettre en date du 6 novembre 1821. Dès les premières lignes et jusque dans le choix des mots et le rythme de la phrase, nous le découvrons tel qu'il nous apparaîtra constamment dans toutes ses "écritures".

" Je n'ai pas voulu écrire à Votre Grandeur avant de connaître un peu la malheureuse paroisse de la Rivière-du-Chêne. M'y voici dans un tourbillon d'affaires et d'occupations infiniment plus délicates et plus occupantes que Votre Grandeur puisse supposer. Il faut être sur les lieux pour cela : voir, entendre et goûter à la misère." La grande préoccupation du jeune curé est évidente : il réclame à cor et à cri un vicaire. "Il y a six à sept cents enfants à préparer à la première communion. Ça paraîtra exagéré à Votre Grandeur mais si on les prend depuis douze à vingt-trois ans, sur une population de près de 6,000 âmes, la proportion se trouve bien gardée... Je vous demande, je vous conjure de me donner pour l'hiver et jusqu'à la première communion un aide et après cela retirez-moi si vous ne jugez pas à propos de continuer vos secours mais au moins que je puisse faire quelque bien et remettre les choses en règle : dans cette paroisse, il y a plus de besoin que dans aucun autre endroit du diocèse."

Pendant vingt-six ans, la correspondance échangée entre le presbytère et les évêchés de Québec et de Montréal fera souvent entendre sinon les mêmes cris du moins les mêmes besoins. Les registres paroissiaux demeurent les témoins irrécusables d'un ministère surchargé. Le nombre des baptêmes atteint facilement les 250 chaque année; les mois de canicule, les sépultures atteignent le nombre des baptêmes. Que dire des épidémies ! La plus effarante, celle du typhus en 1832, devait emporter en moins d'un mois quatre-vingt-dix personnes de tout âge et de toute condition.

Le nouveau curé héritait d'une succession quelque peu compliquée. Des travaux entrepris par son prédécesseur, Messire Jean-Baptiste Gatien, la construction d'un clocher "d'assez mauvais goût pour être obligé dix années plus tard de le faire jeter à terre" et le mauvais état des affaires des anciens syndics qui amènera la mise sous scellés du presbytère construit en 1817, il en aura plein les bras.

\section{Dans l'entourage immédiat: les seigneurs}

Ses premiers rapports avec les messieurs du manoir ne simplifiaient pas les choses. Dès le 11 janvier 1822, une lettre du seigneur Lambert Dumont parvenait à Mgr Plessis se plaignant de la prédication du curé. "Je connais Laviolette, il est capable de l'actionner." Tout 
ceci en marge de déclarations faites par le curé sur la manière de traiter les créanciers et sur les conséquences néfastes de soirées trop tardives.

Le plaidoyer du curé, contenu dans une lettre du 18 février 1822 , à son évêque, nous donne un échantillon de sa rude éloquence et de sa véhémence. "Voici ce que j'ai dit de plus fort. Je ne puis que blâmer hautement ceux qui, ces jours-là, se livrent à la débauche et à l'ivrognerie, ces hommes qui par leurs excès se mettent hors d'état de faire honneur à leurs affaires, de payer leurs dettes, de pourvoir à l'éducation ou à l'éiablissemeni de leurs enfanis, de payer même le salaire du mercenaire... Puis-je excuser des jeunes personnes qui, dans l'âge où l'on est encore sensible aux impressions salutaires de la vertu, abandonnent les fêtes de la religion pour se livrer à des jeux, à des danses, des bals pendant des nuits entières et se mettre par là dans l'impossibilité d'assister aux offices.

"Je n'ai désigné personne, je n'ai dit que ce que je viens d'écrire. J'étais d'un sang-froid imperturbable et je n'avais pas du tout envie de me faire poursuivre en justice; je n'ai parlé ni du village, ni de parti (party).»

Si le curé Paquin fréquentait assidûment la petite aristocratie de son village, il n'avait rien, ni dans son tempérament ni dans son caractère, d'un abbé de salon. Un autre incident, consigné celui-là dans les archives paroissiales, devait mettre, à plus de vingt ans de distance, les mêmes antagonistes en présence. Je cite (le texte est de la main même de M. Paquin): "Pierre Laviolette, écuyer, coseigneur, n'ayant pas le moyen de payer pour sa famille, quoiqu'il eût occupé jusque-là le banc seigneurial en place de la jeune Demoiselle Dumont, seigneuresse, demeurant à Saint-Laurent, s'en vint se mettre dans le banc suivant qu'il occupait déjà pour le seigneur. Monsieur Paquin lui dit que quand il aurait droit au banc seigneurial il ne pouvait en occuper deux.

"Le lendemain, l'honorable G. Roy vint au presbytère, accompagné des Sieurs Globensky, Emm. Ferré, agent et arpenteur du seigneur, et de Laviolette et enfin de M. McKay père. J'observai à M. Roy que nous n'avions pas d'objection à mettre le banc seigneurial suivant la loi injuste de la féodalité, parce que notre fabrique ruinée n'avait pas envie de faire un procès, que Laviolette sans notification au bureau de la fabrique s'était rendu maître d'un second banc. »

\section{Emule du docteur Jacques Labrie, éducateur et historien}

Pour découvrir au curé Paquin un allié naturel, il nous faut donc chercher ailleurs. Il semble bien que des préoccupations identiques, celle de l'éducation d'abord, puis celle de l'histoire, aient rattaché l'un à l'autre le docteur Jacques Labrie et le curé Paquin. Aussi les deux dernières écoles primaires construites par la Commission scolaire de la ville de Saint-Eustache ont-elles été très justement nommées Ecole Jacques-Labrie et Ecole Curé-Jacques-Paquin. 
Le docteur Labrie, "l'un des esprits les plus généreux et peut-être les plus complets de son temps ", avait établi à Saint-Eustache deux écoles supérieures, l'une pour garçons, tenue par M. Paul Rochon, l'autre pour les filles, dirigée par quelques demoiselles. Il les surveillait, les dirigeait en personne, composa pour elles des manuels de géographie et d'histoire canadienne. Son école de filles - devenue par la suite la maison de son gendre, le docteur J.-O. Chénier - était une véritable école normale et les examens publics de ces deux écoles étaient regardés comme autant de fêtes littéraires auxquelles participaient une nombreuse et brillante assistance. La séance de 1826, selon un chroniqueur de l'époque, se déroula en présence de M. le juge Foucher, de Louis-Joseph Papineau, de MM. Neilson et Louis Plamondon, de Québec. Tous ces messieurs, y compris le curé Paquin et le docteur Labrie, célébrèrent l'importance de l'éducation et les mérites des écoles de la Rivière-duChêne.

Nous retrouvons le nom de Jacques Labrie, dans les archives de la paroisse, à l'occasion du choix de cinq paroissiens autorisés à faire rendre compte aux syndics de 1830 au terme de leur dernière année de gestion et comme arbitres à consulter dans les différends qui pourraient s'élever entre les dits syndics en attendant cet intervalle. Son nom figure en tête de liste suivi de celui de MM. de Bellefeuille, J.-B. Laviolette, Eug. Globensky et Jos. Robin.

L'année suivante, soit le 26 septembre 1831, l'aînée des filles du docteur Labrie, âgée de 18 ans, épousait le docteur Jean-Olivier Chénier, de Saint-Benoît. Ce mariage, célébré par le curé Paquin, eut pour témoins les grands personnages du temps, entre autres Louis-Joseph Papineau, Augustin-Norbert Morin, Louis-Michel Viger, Jacques Viger, William Henry Scott, député du comté conjointement avec le docteur Labrie, Côme-Séraphin Cherrier et plusieurs autres. Un mois plus tard, le 29 octobre 1831, les mêmes personnes pour la plupart, entre autres MM. Papineau, Viger et Morin, assistaient, dans l'église de Saint-Eustache, aux funérailles du docteur Labrie, leur ami de cœur, décédé prématurément, à l'âge de 48 ans, d'une pneumonie contractée à l'occasion de la visite des paroisses et des écoles de son comté.

Cette perte était considérable. Dès la session d'automne 1831, la Chambre d'Assemblée, en refusant de voter la liste civile permanente demandée par l'Angleterre, s'engageait dans la voie de l'opposition irréductible. Le projet de publication de l'Histoire du Canada, de Jacques Labrie, après divers atermoiements sera retardé et le manuscrit définitivement perdu lors de l'incendie de la maison Girouard, à SaintBenoît, en décembre 1837.

\section{Relève partielle du curé Paquin, éducateur et historien}

Le curé Paquin, pour sa part, s'offre pour la relève. Il n'exercera jamais, auprès du gendre du docteur Labrie, le docteur Jean-Olivier Chénier, le rôle que le beau-père aurait pu remplir en le dissuadant 
de prendre les armes. Il reprend de plus belle ses illusions d'historien. Il avait rêvé, rien de moins, en compulsant les papiers d'Histoire du Canada de son paroissien, et dans l'admiration de son initiative, que de rédiger une histoire complémentaire de l'Eglise du Canada.

Ses activités pour doter sa paroisse d'institutions scolaires permanentes eurent plus de succès. En 1829, en conformité avec la loi des écoles de fabrique de 1825, une maison d'écoles pour garçons avait été construite au faubourg Saint-Jacques. Par une lettre en date du

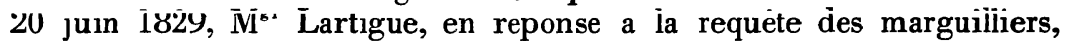
leur permettait de prendre 1,300 livres au coffre de leur fabrique pour une école placée sous le contrôle du curé et des marguilliers. La paroịsse dépensa 2,800 livres et le gouvernement remit 1,200 livres d'après l'acte de 1829 (loi des syndics). En 1833, le nom de Messire Paquin apparaît, à côté de celui de Messire Mignault, curé de Chambly, fondateur du Collège de Chambly, et de celui de Messire J.-C. Prince, directeur du Collège de Saint-Hyacinthe, dans la liste des octrois publics, pour un montant de 100 livres, pour lui permettre d'achever la construction de son école.

Cette année 1833 est une année besogneuse dans la vie du curé de Saint-Eustache. L'augmentation de la population de la paroisse justifiait des agrandissements à l'église. Tous ces projets, longuement discutés depuis 1829 , se réalisent enfin dans la concorde. Une allonge de vingtcinq pieds permettra désormais de loger plus de 240 bancs dans l'église; "l'érection de deux tours prolongeant les murs latéraux cacheront désormais la défectuosité du vieux corps de l'église trop bas pour la régularité des proportions et lui donneront une apparence plus digne du Dieu qu'on y adore. " (Requête de 1830.)

Afin de faire accepter avec plus d'empressement ce projet, le curé s'engageait à faire construire un couvent à ses propres frais. Construit en 1833 , détruit en 1837, reconstruit en 1838 , ce couvent ne devait être occupé par les Sœurs de la Congrégation qu'en 1849, deux années après la mort du curé.

\section{Messire Jacques Paquin, archiprêtre}

Déjà, dès 1824, $\mathrm{M}^{\mathrm{gr}}$ Lartigue, évêque à Montréal, proposait à l'évêque de Québec le nom de Messire Jacques Paquin comme archiprêtre. La nomination ne devait venir que le 8 février 1833, signée par $\mathrm{M}^{\mathrm{gr}}$ Signay. Le nouvel archiprêtre se voit confier la bonne marche de diverses affaires : l'érection canonique des paroisses de Saint-Benoît, Sainte-Scholastique et Saint-Colomban; des enquêtes canoniques au sujet de la construction ou de la réparation d'églises et de presbytères, à Sainte-Thérèse (1833), à Sainte-Scholastique (1834), à Saint-Hermas, à Sainte-Rose, à Saint-Jérôme des Mille-Iles (1835), à Sainte-Geneviève (1836). Un projet de journal ecclésiastique, auquel $\mathrm{M}^{\mathrm{gr}}$ Lartigue semblait donner son assentiment, fut définitivement écarté en 1832 par $\mathrm{M}^{\mathrm{gr}}$ Panet et $\mathrm{M}^{\mathrm{gr}}$ Signay. 


\section{Prodromes d'insurrection}

Pendant ce temps les nuages et les divisions politiques commençaient à s'accumuler. J'ai retrouvé dans la correspondance de $\mathbf{M}^{\mathrm{gr}}$ JeanJacques Lartigue, en date du 12 décembre 1834, la lettre suivante qui indique clairement la présence à Saint-Eustache de deux groupes bien distincts. La lecture de cette lettre offre le double intérêt de nous renseigner sur l'attitude que préconise l'évêque en pareil cas et sur les responsabilités qu'il désire voir assumer par ses prêtres.

"Je vous renvoie ci-incluse la demande que vous ont faite par écrit plusieurs habitants de votre paroisse sur la teneur de l'annonce d'une messe qu'ils voudraient faire chanter. Il est évident que vous ne pouvez annoncer cette messe en la manière qu'ils proposent sans choquer les sentiments de plusieurs de leurs co-paroissiens et sans déshonorer votre ministère par l'assomption patente d'un parti en politique. Il vous est facile de faire sentir aux intéressés que si vous adhériez à leur demande, dès le lendemain ceux du parti opposé auraient droit de vous demander à leur tour l'annonce absolument contraire d'une autre messe, et qui serait conforme à leurs prétentions politiques, sans que vous puissiez raisonnablement les refuser, puisque vous n'êtes pas juge entre les deux partis. La seule annonce que je permettrai en pareille circonstance serait celle-ci : Tel jour, à telle heure, nous chanterons dans cette église une messe d'actions de grâces, demandée par plusieurs particuliers de cette paroisse. Mais s'ils ne veulent pas de cette manière, restez tranquille.

"P.-S. Autre chose. Dans un cas aussi clair que celui de l'annonce maladroite qu'on vous a demandée à faire dans votre église et que vous aviez déjà déclaré justement aux requérants être impensable, pourquoi compromettre inutilement votre supérieur en provoquant une réponse de sa part sur cette affaire? Les supérieurs ne doivent être mis ainsi en avant que dans les cas douteux et qui sortent de la voie ordinaire, pour vous appuyer de leur autorité quand les plaignants se sont directement adressés à eux. Hors de là c'est leur donner de l'odieux à pure perte dans une chose qui vous regarde personnellement. "

\section{J.-J. Lartigue}

12 décembre 1834, - Lettre à Jacques Paquin, archiprêtre.

\section{De l'insurrection des esprits à l'insurrection armée}

"Ne pas être juge entre les deux parties!" Le curé Paquin en était-il vraiment capable? Son tempérament sanguin fort prononcé l'entraînait souvent à des violences de paroles et les positions qu'il avait prises pendant les années de luttes politiques ne lui facilitèrent pas la tâche de modérateur au moment de l'insurrection armée.

Au regard de l'histoire Messire Jacques Paquin fait donc quelque peu figure de "bureaucrate "; il faut dire que la présence et l'activité de son « patriote » voisin, l'abbé Etienne Chartier, curé de Saint-Benoît, invitent et inclinent les esprits à un classement peut-être trop sommaire. 
Pour sa part, $\mathrm{M}^{\mathrm{gr}}$ Lartigue profita du banquet du sacre de $\mathrm{M}^{\mathrm{gr}}$ Bourget pour donner à son clergé des directives précises : "Dans les circonstances où se trouve le pays, les pasteurs doivent faire tous leurs efforts pour rétablir la charité et l'union parmi leurs ouailles; ils doivent représenter à leurs paroissiens qu'il n'est pas permis de se révolter contre l'autorité légitime, ni de transgresser les lois du pays; ils ne doivent pas absoudre dans le tribunal de la pénitence ceux qui enseignent de violer ces lois. » L'autorité religieuse fit suivre cette déclaration de quelques lettres aux curés de Saint-Benoît et de Saint-Eustache, leur disant : au premier, la peine que lui cause sa conduite peu conforme aux principes de l'Eglise; et au second, de faire comprendre à son voisin, le curé de Saint-Benoît, la terrible responsabilité qu'il accumule sur ses épaules. "J'ai ressenti un profond chagrin, écrit-il à M. Chartier, en voyant que vous vous mettiez au blanc. Vous seriez étonné d'apprendre ce qu'on vous impute, et quels sont les propos que l'on vous fait tenir. En attendant que je puisse librement et amicalement converser avec vous là-dessus, je vous conjure de vous retirer de cette mêlée et de considérer que vous avez une autre espèce de guerre à faire plus glorieuse et plus en harmonie avec les services de votre saint état. » (10 juillet 1837)

L'abbé Emile Dubois, l'historien du "Feu de la Rivière du Chêne ", note quelques-uns des nombreux et réels excès auxquels se livrèrent les patriotes du camp de Saint-Eustache. Monsieur Paquin lui-même les signale dans ses écrits et $M^{\mathrm{gr}}$ Lartigue dans une lettre à l'évêque de Québec parle, lui aussi, "des vagabonds de Saint-Benoît, de SainteScholastique et d'une partie de Saint-Eustache qui pillent et volent partout ". "Mais je pense, ajoute-t-il, que si les troupes ne vont pas les attaquer chez eux, les surveillant seulement de loin et les laissant se consumer d'eux-mêmes, ils seront bientôt débandés."

La désertion du camp de Saint-Eustache, à certains jours du début de décembre, sous la seule influence de M. Paquin, donne raison à l'évêque de Montréal. Mais l'armée anglaise, sous les ordres de Colborne, est déjà rassemblée pour la journée sinistre du 14 décembre 1837.

\section{"Pastor invictus》 - On reconstruit}

Nous pouvons difficilement concevoir, à cent vingt-cinq ans de distance et dans le contexte d'un monde si différent d'autrefois, l'ampleur de la consternation qui suivit toute cette tragédie. Désolation temporelle; désolation spirituelle tout aussi bien. Pour le curé Paquin et pour ses paroissiens, tout était à refaire. Il s'emploie d'abord au plus pressé : faire cesser la violence et le brigandage. "Les gens de Gore et de Chatham sont coupables de grande rapine ", devait écrire $\mathbf{M}^{\mathrm{gr}} \mathrm{Lar}$ tigue. La célébration de la messe se fait dans des maisons d'écoles et des maisons particulières. Il faut également desservir la paroisse de Saint-Benoît, victime d'un vandalisme éhonté et privée de son pasteur. Déjà surgit dans la pensée du curé Paquin la volonté d'obtenir les indemnités nécessaires à la reconstruction. La publication du « Journal historique des événements arrivés pendant la rebellion du comté des 
Deux-Montagnes, depuis les soulèvements commencés à la fin de novembre jusqu'au moment où la tranquillité fut rétablie " (opuscule de 96 pages, attribué parfois à $M$. Desève, son vicaire) s'inspire visiblement de cette intention : obtenir du Gouvernement une indemnité, en montrant que bien peu de ses paroissiens s'étaient ralliés aux Patriotes et que ceux-ci avaient été leurrés par des chefs étrangers pour la plupart à notre nationalité.

L'examen des comptes publics de la fabrique nous révèle l'extrême minutie du curé comptable : le montant de la vente des bancs pour l'année 1837 est diminué du vingt-quatrième. Dès 1838, le couvent est reconstruit afin de permettre son utilisation comme chapelle. Une souscription par petites sommes, de vingt sous à un écu, rapporte 216 livres et six sous. Le curé pour sa part défraie le coût de tout le bois de charpente.

La reconstruction de l'église accuse un retard. Les projets de division de la paroisse et l'érection subséquente de la paroisse de SaintAugustin en sont la cause. Messire Charles Ducharme, curé de SainteThérèse, est chargé de cette desserte avant la nomination de son premier curé, l'abbé Desève, vicaire de Saint-Eustache. Ce fut l'un des moments les plus pénibles de la vie du curé Paquin. La correspondance échangée à cette époque avec $\mathrm{M}^{\mathrm{gr}}$ Bourget nous révèle une situation tendue. Dans une lettre en date du 5 avril $1841, \mathrm{M}^{\mathrm{gr}}$ de Montréal lui fait part de plaintes reçues : "... il ne prend pas soin du cimetière et même de l'ancienne église abandonnée aux animaux; il laisse les enfants de chœur jouer, rire, se bousculer pendant les offices religieux; enfin il prêche rarement, bien qu'il ait de la facilité pour le ministère de la parole. " Il le conjure de mieux s'acquitter de ses devoirs envers sa paroisse «qui a déjà été désolée au temporel sans souffrir de la désolation spirituelle».

Pourtant, dès le 27 décembre 1840 , par un acte notarié, le curé Paquin avait consenti un emprunt de 10,000 livres (ancien cours) à la fabrique, pour assurer la restauration de l'église. Le 30 septembre 184l, dans une lettre au curé Paquin, $\mathrm{M}^{\mathrm{gr}}$ Bourget se réjouit de l'achèvement de l'église qu'il viendra bénir le 14, octobre. "Fasse le ciel que cette solennité console vous et vos paroissiens après tant et de si grandes tribulations par lesquelles vous êtes passés."

Une question ici se pose : quelle a été l'ampleur de cette restauration et partant des dégâts causés par l'incendie ? Il arrive souvent que des visiteurs, et même des paroissiens nous posent des interrogations relativement à la présence d'éclats de pierre causés par les balles anglaises sur la façade de l'église actuelle. A cela nous pouvons répondre d'abord en rappelant que les deux dates 1833-1843 sont inscrites sur le fronton de l'église, que le livre des comptes de la fabrique, pour 1841, indique le montant versé "pour couvrir la vieille église et les tours ", enfin que l'inventaire des biens, meubles et immeubles de la Fabrique de Saint-Eustache, en date du 10 juillet 1845, tel que préparé par le curé Paquin, se lit comme suit : "Une église en pierre bâtie en 1782 par M. Perrault. Allongée de 25 pieds, deux tours surmontées 
de deux clochers à deux lanternes couverts en fer blanc avec coupoles et flèches. La façade du portail et des tours est en pierre de taille de deux entablements d'ordre grec ionique et dorique par M. Paquin, curé, en 1833, brûlée par le général Sir John Colborne, surnommé le vieux "Bruleau ", 14 décembre 1837; rebâtie et améliorée sous la direction du même curé (1840) et achevée, quant au dehors en 1845. Cette église a été estimée à $L$. 7,000 avant sa destruction, elle en vaut mieux maintenant quant à l'extérieur ${ }^{1}$. »

\section{Les dernières années}

En 1843, le curé Paquin, curé bâtisseur s'il en fût, cède la place de nouveau à cet autre personnage, celui que le R.P. Thomas-Marie Charland, o.p., s'est appliqué à nous faire connaître, qui rêvait, rien de moins, que de devenir grand historien. Le 12 janvier, $\mathrm{M}^{\mathrm{gr}}$ Bourget, par lettre, lui permet de faire le tour du diocèse et d'aller dans celui de Québec pour faire des recherches dans le but, affirme-t-il, de donner une Histoire ecclésiastique du Canada "que vous exécuterez d'une manière honorable pour la religion et pour votre pays ". C'est alors, pour Messire Jacques Paquin, un moment d'euphorie. Mais bientôt c'est le rappel à des nécessités plus urgentes et... le rappel tout court, signifié d'abord par un billet du 29 août. L'évêque de Montréal explicite davantage son geste dans une lettre du 28 septembre : "Avant tout le royaume des cieux, pensez-y. Car vous serez jugé non pas sur vos qualités d'historien, philosophe, littérateur, mais sur vos devoirs de pasteur. "

Le curé Paquin en conservera autant d'entêtement que d'amertume. Il récapitule lui-même, pour notre information, dans une longue lettre à son évêque, toute cette "petite histoire ":

"Cette intimation de ne pas négliger ma paroisse semble m'interdire le temps et les moyens de faire imprimer mon ouvrage : car il ne me reste rien d'autre chose à faire. Ce qui paraît confirmer cette entente, c'est d'abord mon rappel précipité de Québec, en 1843, ensuite les recherches incessantes que fait, depuis cette époque, le P. Martin sur l'Eglise du Canada, puis encore les extraits faits furtivement chez moi et en mon absence sur des brouillons... Votre grandeur m'avait permis en 1843 de prendre tout le temps que je voudrais pour compléter mes recherches historiques dans les diocèses de Québec et de Montréal. Ai-je négligé pour cela le soin de ma paroisse? N'est-ce pas moi qui ai fait relever de leur cendre l'église, presbytère, couvent et les édifices paroissiaux de Saint-Eustache et ai même conduit tous les travaux, malgré la misère de ma pauvre paroisse ruinée par le pillage, la conflagration et le morcellement de la meilleure partie de son territoire en opposition aux réclamations de plusieurs des habitants qui passent encore à travers leur ancienne paroisse pour aller dans celle

1 En janvier 1853, la Commission d'Indemnité aux victimes de 1837 accordait à la Fabrique de Saint-Eustache, le montant de 2,312 livres, 10 sh. (cours actuel du pays). 
qu'on leur impose de force? J'ai fait bâtir plus de six maisons d'écoles de fabrique et mis sur pied onze écoles catholiques que je surveille seul. N'ai-je pas fait une foule de voyages, de frais, de missions, dans une des côtes de ma paroisse pour en éloigner les misérables suisses ? Dans le moment même, j'y fais ériger une chapelle pour cette fin. Ai-je besoin de dire que j'exerce les autres fonctions du ministère curial ? que je fais les frais des retraites, etc. Pourquoi donc cet ultimatum, cette allusion de philosophe, ces menaces et enfin ce revirement contre moi ? Je sais bien, Monseigneur, que je suis un pauvre misérable sans recommandation, mais ce n'est pas en ravalant, en avilissant ou dégradant un homme qu'on en fait quelque chose car l'opprobre avilit l'âme et flétrit le courage."

Et $\mathrm{M}^{\mathrm{gr}}$ Bourget de répondre (30 mai 1845): "Tout ce que j'ai à vous demander et à exiger de vous c'est que vous ne négligiez pas le soin de votre paroisse pour le désir de donner au monde une nouvelle histoire. »

Le 19 octobre 1847, Messire Jacques Paquin rédigeait et signait le dernier acte qui figure dans les archives paroissiales de Saint-Eustache. Le mardi 7 décembre 1847, à 3 heures p.m., il décédait à son presbytère, à l'âge de 56 ans. Il fut inhumé en dessous du sanctuaire de son église paroissiale, le 13 décembre suivant. Monseigneur Bourget et le personnel de l'évêché étaient en retraite; seuls quelques curés des paroisses voisines - Sainte-Rose, Saint-Benoît, Saint-Martin, SaintAndré - ont signé l'acte de sépulture.

Monsieur Louis-Edouard Bois, dans son Garde-note historique (déposé aux Archives du Séminaire de Nicolet) a rédigé sous cette forme la notice qu'il consacre au curé Paquin. "C'était un homme d'une grande activité. Son infatigable énergie le portait à s'occuper de tout. Ce serait une injustice néanmoins à la mémoire de ce prêtre laborieux de juger de ses essais littéraires d'après le progrès de la science actuelle plutôt que d'après l'état de la littérature à l'époque où il traçait laborieusement ses vues.

"Disons toutefois que promoteur zélé, intelligent et éclairé de l'éducation et aussi de l'agriculture, il a laissé des traces de ce que pouvaient dans ces temps difficiles des hommes dévoués."

Une inscription gravée sur granit a été apposée sur le mur latéral ouest de l'église de Saint-Eustache. Ce geste fut provoqué, semble-t-il, par l'idée du monument Chénier, souscrit en grande partie par la population du comté des Deux-Montagnes et érigé au carré Viger, à Montréal. C'était aux jours fiévreux de l'affaire Riel. Jusque dans sa tombe - trente-huit ans après son décès - la mémoire d'un prêtre, Messire Jacques Paquin, qui fut d'abord un curé de paroisse actif et zélé, risquait d'être compromise en l'opposant indûment aux champions imprudents, mais sincères, de nos libertés politiques.

$\mathrm{M}^{\mathrm{gr}}$ Louis-Joseph Rodrigue, P.D. curé $\left(13^{\mathrm{e}}\right)$ de Saint-Eustache, P.Q. 